\title{
La construcción social de la violencia
}

\author{
Miquel Domènech i Argemí y Lupicinio Íñiguez Rueda \\ Universitat Autònoma de Barcelona
}

La familia es uno de los contextos donde la violencia se produce de una forma más espectacular, alarmante, inesperada y cruel. Todas las combinaciones son posibles y de ello dan testimonio diario los medios de comunicación. Padres y madres contra hijos, miembros de la pareja unos contra otros. Hermanos contra hermanos e hijos contra padres. Puede parecer que se trata de un fenómeno moderno dada la repercusión que tal tipo de sucesos ha tomado, pero sabemos bien que se trata de algo tan antiguo como la familia misma y sus huellas se pueden rastrear en la producción literaria clásica y moderna que, de hecho, constituye una buena parte de nuestra memoria histórica. La familia de Pascual Duarte, Los hermanos Karamazov, son ejemplos del tipo de obras que alimentan ese imaginario colectivo que muestra a la familia como un locus de tensión y violencia.

Ahora bien, es cierto que la cuestión de la "violencia familiar" es, entendida como problema social, una construcción reciente. Han hecho falta una serie de factores sociohistóricos para que una agresión en el contexto familiar sea etiquetada como un caso de violencia familiar, de la misma manera que haría falta otro tipo de contexto sociohistórico para que la violencia familiar fuera vista como una forma de violencia política.

La importancia del contexto se pone de manifiesto cuando constatamos que no todas esas formas de violencia que comentábamos más arriba son igualmente probables en nuestra sociedad actual. Por ejemplo, ¿qué es mas probable, que una mujer agreda a su compañero o que éste agreda a aquélla? ¿qué es más probable, que hijos o hijas agredan a sus padres o viceversa? ¿que las hermanas agredan a sus hermanos a al revés? Hay un patrón en esas agresiones que no parece adaptarse a las probabilidades esperadas. Cada uno/a puede agredir a cualquier otro/a, sí, es cierto. Pero las cosas suceden de manera distinta la mayor parte de las veces, siguiendo patrones y normas que consiguen regular la dirección de la violencia y que constituyen la marca de un tipo de sociedad concreta.

Como una aportación más a la inteligibilidad de la violencia, vamos a presentar aquí algunas aportaciones que ha realizado la Psicología Social al entendimiento de los comportamientos agresivos y de la violencia social. Lo hacemos desde nuestra ubicación disciplinar y académica porque es la que conocemos mejor, pero también porque nos va permitir ilustrar cómo articular procedimientos de análisis desde perspectivas bien distintas y con enfoques diferentes. $Y$, finalmente, porque nos da pie a ilustrar las influencias que ejerce el contexto social, histórico e ideológico sobre la comprensión de cualquier proceso social como la agresión y la violencia. 


\section{La agresividad / el comportamiento agresivo tal y como}

\section{ha sido tratado en la Psicología social convencional}

El análisis que ha realizado la Psicología Social convencional de la agresión y la violencia, se puede dividir en dos etapas: el que realizaron las perspectivas más clásicas y el que se ha realizado más contemporáneamente.

\subsection{Las perspectivas clásicas}

El estudio del comportamiento agresivo en la Psicología Social convencional atraviesa completamente el espacio que media entre su concepción como algo interno, a su concepción como algo dependiente de fuerzas externas. Las primeras explicaciones son mayoritariamente de naturaleza instintivista y las últimas de naturaleza ambientalista.

\section{a) Teorías instintivistas}

Las teorías y modelos instintivistas son los que mejor reproducen la necesidad de explicar este tipo de comportamientos desde el interior de la persona. En efecto, tales modelos identifican una causa del comportamiento agresivo que se sitúa dentro de la persona. Esta causa es, para este tipo de explicación, un instinto. La conexión con el darwinismo y con todas las demás teorías psicológicas y sociales que estuvieron afectadas por él, son claras.

En esta perspectiva, la agresión se desencadenaría de manera inevitable ante la aparición de una señal. Sus funciones serían las de la supervivencia de la especie y las posibilidades de modificación muy escasas (Eibel-Eibesfeldt, 1973; Lorenz, 1963). En Psicología existen muchas explicaciones adaptadas a este modelo simple. Probablemente la más conocida de todas sea el psicoanálisis que prevé el mismo tipo de explicación para estos comportamientos aunque añadiéndoles una función constitutiva de la personalidad humana, es decir, se trata de una redefinición funcional del instinto agresivo (Freud, 1930).

Las consecuencias sociales, políticas e ideológicas de este tipo de explicación son claras. Sobretodo si tenemos en cuenta el carácter inevitable del instinto y del comportamiento que programa. La introducción de modificaciones en este esquema es imposible, o muy difícil, con lo que las dependencias de variables más sociales o relacionales parece imposible, tanto en el plano teórico como de intervención social. En Psicología ha cristalizado, además, en una noción poco inocente cual es la de personalidad agresiva (Muñoz, 1988).

\section{b) Fustración-agresión}

Como resultado de las teorías psicoanalíticas y de la importante emergencia de los modelos ambientalistas, surge una explicación intermedia, a caballo entre lo interno y lo ambiental. Se trata de la llamada "teoría de la frustración agresión". En esencia, este modelo prevé que, efectivamente, la agresión es un comportamiento resultante de una pulsión interna pero que esta pulsión depende de un elemento externo: la generación de frustración. En definitiva, cuando las personas ven impedida la acción que pretenden por alguna fuerza externa, experimentan frustración. Su aumento, 
desencadena agresión. La agresión elimina la frustración y no se producirá nuevamente hasta que los niveles de frustración sean nuevamente altos (Dollard et al., 1939)

El ejercicio de aunar elementos de naturaleza interna y externa en la explicación de la agresión ha sido social y teóricamente muy interesante. En efecto, por una parte constituye uno de los poco ejemplos de integración o intento de integración de teorías prácticamente irreconciliables. Por otra, conecta con la concepción cotidiana más extendida de que la gente puede acumular frustraciones y aguantar la agresividad que le produce sólo hasta cierto punto. No es éste el lugar, pero invitamos a una reflexión sobre qué habrá producido qué, es decir, si será que esta teoría ha posibilitado esta concepción cotidiana de la agresión o, por el contrario, habrá sido esta concepción la que ha creado esta teoría.

\section{c) Aprendizaje social}

Las teorías del aprendizaje social son las resultantes de la dominación del positivismo en psicología. En nuestra disciplina se le conoce con el nombre de Conductismo, pero hay que entender que se refieren a la misma cosa. En las teorías del aprendizaje, el elemento constitutivo de los comportamientos proviene de la asociación de una determinada respuesta a un estímulo concreto, asociación que queda cristalizada por el refuerzo de la ejecución de ese comportamiento.

Para el caso del comportamiento agresivo el mecanismo es idéntico. Ciertos estímulos provocan cierta clase de comportamientos agresivos, los cuales son permanentes por el refuerzo que se ha producido de sus ejecuciones tras cada una de las apariciones del estímulo.

En Psicología Social la más importante teoría de todas las que se refieren al comportamiento agresivo es la del "Aprendizaje social" (Bandura, 1976; Bandura y Ribes, 1975). En esencia, lo que esta teoría postula es que se 'pueden aprender comportamientos por imitación, más concretamente, por la visión de que esos comportamientos observados han sido recompensados o reforzados. De este modo, si alguien ve que un comportamiento agresivo de una persona es reforzado, entonces lo puede aprender.

El trabajo de Bandura ha sido extraordinariamente fecundo en la comprensión del comportamiento agresivo, sobretodo porque ha permitido distinguir analíticamente el aprendizaje de un comportamiento y su ejecución. Es decir, se puede efectivamente aprender un comportamiento agresivo porque se ha visto cómo resultaba recompensado en otra persona, pero eso no implica que se tenga que ejecutar. Se ha aprendido, se conoce, se sabe cómo ejecutarlo, pero puede que se realice o puede que no. La principal influencia de este modelo se ha dejado sentir en el estudio de los efectos de la visión de violencia. Más concretamente, la teoría permite introducir elementos de inteligibilidad menos grotescos que los habituales, al discutir si las escenas violentas que podemos ver en el cine o en la televisión comportan, inevitablemente, un aumento de dichos comportamientos. La respuesta es muy clara desde el punto de vista de Bandura, son comportamientos que se aprenden, efectivamente, pero su aprendizaje dista mucho de su ejecución y, por lo tanto, pueden ser realizados o no dependiendo a su vez de otros tipos de aprendizajes (Bandura, Ross y Ross, 1963) 


\subsection{La aportación de la Psicología Social contemporánea (aunque convencional)}

La psicología social contemporánea ha introducido nuevos elementos de inteligibilidad a la agresión que se desmarcan tanto de las explicaciones de naturaleza interna como de las de naturaleza externa. Más específicamente, introduce el contexto relacional en el que se produce todo comportamiento agresivo y violento. La agresión es siempre contra alguien y es ejecutada por alguien. Si no media relación, cualquiera que sea su naturaleza real o simbólica, la agresión no puede tener lugar (Muñoz, 1990)

Esta explicación tiene las siguientes características, claves para poder definir un acto como agresivo:

- La ubica en un contexto de interacción interpersonal o intergrupal

- Es considerada como una acción intencional (la intencionalidad es un elemento clave en la definición de un acto como agresivo)

- El daño, como consecuencia del acto agresivo

Sin embargo, ninguno de estos elementos, ni por separado, ni conjuntamente, permiten aún decidir si un acto es o no agresivo. Para poder definirlo como agresión hay que incorporar la noción de norma. La agresión es, pues, por encima de todo un comportamiento antinormativo.

Esto implica que en un contexto de interacción interpersonal y/o intergrupal, sea necesaria la realización de una atribución de agresividad, o dicho de otro modo, actor, víctima y/o observador tienen que hacer una atribución de este tipo para que puedan concluir que están ante un acto agresivo.

Este tratamiento de la agresión deja aún algunos problemas en el aire. Por ejemplo, ¿cómo poder entender la agresión al margen del ejercicio del poder? ¿cómo entenderla fuera del contexto de las relaciones de dominación? La agresión es dominación o intento de dominación, es ejercicio de poder coercitivo.

Al ubicar la agresión en un contexto interpersonal o intergrupal y normativo, y necesitar una definición o identificación como agresión, se está poniendo de manifiesto que "agresión" es una palabra que no está relacionada necesariamente con observables, sino que está conectada con otras formas lingüísticas. Esto nos lleva directamente al ámbito de los discursos y del análisis estos (Gergen, 1984).

El tipo de análisis convencional que hemos esbozado hasta ahora desvincula el comportamiento agresivo del mantenimiento del orden social. Goza, en esas versiones, de una independencia prácticamente absoluta con respecto a la estructura y órdenes sociales. La única presencia que "lo social" tendría, sería en tanto que "contexto social" que en ocasiones afecta al comportamiento agresivo o a la violencia (por ejemplo si la violencia en los medios de comunicación aumenta o no el comportamiento agresivo). Veamos con más detalle, qué supuestos están en la base de estas explicaciones convencionales y cuál es el tipo de consecuencias que acarrean. 


\section{Supuestos sobre la agresión que están implicados}

A partir de este somero repaso que hemos realizado a las principales aportaciones que la psicología social ha hecho al estudio del comportamiento agresivo es posible discernir algunos supuestos que subyacen a la práctica totalidad de los planteamientos. Nos vamos a referir básicamente a tres que fueron ya identificados hace unos años por Lubek (1979):

1) Se asume que la violencia y la agresión deben ser explicadas a nivel individual o, incluso, intraindividual. Se deja de lado, por tanto, el papel de los grupos mayores o las instituciones. Ello lleva a una prevalencia de los estudios de laboratorio basados en el paradigma Estímulo-Respuesta.

2) Se asume que la violencia es perjudicial para la sociedad y se identifica como violento aquello que atenta contra el poder establecido. Se descartan preguntas acerca del efecto de las acciones violentas a largo plazo.

3) Se asume que controlar, reducir y reprimir la violencia es un objetivo válido en cualquier caso. Por otra parte, dado que se entiende que la investigación científica es "neutral", se defiende que pueda ser usada por cualquier instancia que quiera promover el bien social. Según Lubek, sólo hay que examinar la realidad histórica y comprobar quién se aprovecha y para qué de los resultados de las investigaciones para darse cuenta de que eso no es más que una falacia.

Quisiéramos subrayar, por nuestra parte, hasta qué punto estas explicaciones convencionales, al abordar la cuestión de la agresividad bajo estos supuestos, evitan cualquier tipo de cuestionamiento del orden social imperante. No sólo eso, de hecho, puede decirse que, tal y como señalan StaintonRogers et al. (1995), en realidad cumplen un papel reforzador del status quo a través de un discurso ideológico que muestra dos caras diferentes según convenga. Por un lado, cuando la atención se centra en la agresión como problema individual, se evita plantear la cuestión en términos políticos. Por otra parte, si, contrariamente, la atención recae sobre la agresión como conducta socialmente desviada, se evita plantear la cuestión de la violencia institucional ya que se vincula la violencia o la agresión con la actividad de grupos desviados o con el efecto de aprendizajes disfuncionales, pero no se hace referencia a las acciones violentas que el poder institucionalizado lleva a cabo. Y sin embargo, si hay algún problema, si se requiere alguna solución, aquél o ésta tienen que ver con la acción de algún tipo de poder regulador sobre aquellos grupos o individuos que muestran una conducta socialmente inadecuada.

Así pues, el punto de vista sobre el que la psicología social convencional se asienta, a pesar de, o precisamente por, su declarado aposicionamiento político, se muestra claramente como un dispositivo ideológico que cumple una función social reguladora en absoluto ajena al ejercicio del poder. De ahí la necesidad, resaltada por los que compartimos una visión crítica de la práctica de la psicología social (Domènech e lbáñez, 1998; Ibáñez e Îñiguez, 1997), de introducir una reflexión política que no esté enmascarada y que sitúe a la ciencia social en una posicón definida. Dicho de otro modo, y tomando para ello las palabras de Ignacio Martín-Baró --psicólogo social y religioso jesuita asesinado en El Salvador por decir cosas como la que siguen:

"...la misma formalidad del acto como violento o agresivo supone una definición social sin la cual se pueden confundir los golpes percibidos por un espectador en una aglomeración pública con los golpes recibidos por un preso político a manos de sus captores policías. En este sentido, la perspectiva de la psicología social como estudio de la acción en cuanto ideológica nos obliga a ir más allá de la apariencia 
visible de la conducta y penetrar en sus raíces históricas, tanto por lo que tiene de expresión de unas estructuras sociales como por los intereses de clase que la persona o grupo involucrados ponen en juego" (Martín-Baró, 1983:370).

\section{El caso de Kitty Genovese}

Para ilustrar la importancia que tiene el contexto de interpretación en la determinación de lo que se toma o no como conducta violenta seguiremos el magnífico relato vivencial que Frances Cherry hilvana en The 'stubborn particulars' of Social Psychology acerca del papel que tiene una disciplina científica en ese proceso de dilucidación.

Tal relato gira en torno al proceso de transformación que esta autora experimentó en su manera de percibir un tristemente célebre suceso que sirve a gran cantidad de manuales de Psicología Social para ilustrar los determinantes que intervienen en la conducta altruista. Nos referimos, por supuesto, al asesinato, el 13 de mayo de 1964, de una joven neoyorkina llamada Kitty Genovese.

Kitty Genovese recorría una calle del acomodado barrio neoyorkino de Queens poco después de las 3:00 AM. cuando fue violada y asesinada por un hombre a pesar de los gritos de auxilio que profirió y de que hasta treinta y siete personas pudieron contemplar desde sus ventanas el desarrollo de los acontecimientos. Nadie intervino, ni tan sólo para llamar a la policía, hasta mucho tiempo después de iniciado el asalto, cuando ya era demasiado tarde. El caso de Kitty Genovese mereció extensos análisis e interpretaciones que fueron apareciendo en el New York Times a lo largo de los dos meses posteriores a su muerte y consagró a dos jóvenes psicólogos sociales Bibb Latané y John Darley. Latané y Darley (1970) hicieron del análisis de la trágica muerte de Kitty Genovese el núcleo de su teorización acerca de la conducta de facilitación de ayuda.

Ahora bien, Cherry (1995) se plantea algunas preguntas: ¿Por qué razón el asesinato de Kitty Genovese se convirtió en un ejemplo para ilustar la temática relacionada con las conductas sociales en las que se requiere la 'intervención de los testigos'? ¿Qué proceso de construcción de significado se puso en marcha? Y ella misma nos hace notar que para responder tales cuestiones es preciso, en primer lugar, tener en cuenta el contexto cultural de la América de los años sesenta. Un contexto en el que los miembros de esa cultura no acostumbraban a hacer gran cosa para intervenir en casos de violencia dirigida hacia mujeres. Como señala esta autora, detalles como éste no formaban parte del "proceso de abstracción/construcción" por el que los "procesos generales" de la conducta social fueron hipotetizados y posteriormente comprobados. En ese momento, mediados de los sesenta, y en ese lugar, norteamérica, el suceso fue abstraído como un caso en el que hubo una emergencia y nadie intervino para ayudar.

Ahora bien, ¿es posible contemplar ese suceso de una manera diferente? Si transformamos el contexto de interpretación, definitivamente sí. Cherry (1995) cuenta la importancia que tuvo el texto de Brownmiller (1975) para que, en su caso, ello fuera posible. Brownmiller relata con detalle la violación y muerte de Kitty Genovese en su libro dedicado a la temática de la violación de mujeres. $\mathrm{Al}$ tomar el caso de Kitty Genovese, ésta última autora pone sobre la mesa los condicionantes particulares relativos a cuestiones de género que están implicados en este incidente. Ese detalle fue suficiente para que, a mediados de los ochenta y en un contexto social definitivamente sensibilizado respecto a las agresiones a mujeres, Cherry pudiera plantearse un mismo hecho en unos términos absolutamente diferentes a los que hasta ese momento había utilizado. La cuestión de la prestación 
de ayuda en situaciones de emergencia pasaba a un segundo plano y, en su lugar, emergía claramente la cuestión del maltrato a las mujeres. La muerte de Kitty Genovese se había convertido en un nuevo caso de violencia hacia una mujer perpetrado por un hombre en el que nadie había intervenido para prestar ayuda. El contexto cultural había cambiado y, con él, el nombre con el que denominar el caso de Kitty Genovese.

Pero eso había sido mucho tiempo después del incidente. Ya hemos dicho que inmediatamente después de su enorme eco en los diarios se había convertido en un caso paradigmático de los condicionantes que intervienen en la prestación de ayuda en situaciones de emergencia. Es en este sentido que dio origen a toda una saga de experimentos supuestamente inspirados en ese hecho verídico. Una persona que sufría un ataque epiléptico, una habitación que se llenaba de humo, una secretaria que se caía de una silla... eran algunas de las situaciones experimentales que se diseñaban para medir la disposición a ayudar de los testigos presenciales. Curiosamente, sin embargo, y tal y como Cherry (1995), una vez más, destaca:

"la violencia sexual/de género había sido excluída de la fase de abstracción de hipótesis acerca de la realidad social (...) el ataque a una mujer dejaba de ser un componente esencial en la exploración de laboratorio de lo que siginificaba el acontecimiento" (p.21)

Cherry (1995), no obstante, menciona también los dos únicos experimentos que, aunque surgidos también en ese mismo campo temático de la dispensación de ayuda en situaciones de emergencia, de alguna manera sí que introducen la cuestión del género.

Borofsky et al. (1971) realizaron un estudio en el que díadas formadas por hombres y mujeres simulaban pegar a hombres o mujeres, según las cuatro combinaciones posibles, en presencia de obsservadores. Resulta enormemente significativo que, en la condición en la que un hombre pegaba a una mujer, ninguno de los seis observadores hombres intervenía para impedirlo, mientras que la ayuda a otro hombre era relativamente frecuente.

Shotland y Straw (1976), por su parte, encontraron en otra serie de experimentos que la intervención era mucho más frecuente cuando los observadores percibían a atacante y víctima como extraños (65\%) que cuando los creían casados (19\%). Pero lo más interesante es la conclusión a la que llegaban: después de señalar lo bueno que sería que los vecinos se conociesen mejor para no caer así en falsas apreciaciones y mejorar el control social en la comunidad,afirmaban:

"si pudiéramos conseguir tal control en lo que se refiere al caso de un hombre pegando a una mujer, seríamos capaces de restringir la victimización de las mujeres a sus maridos o allegados más próximos" (p.999).

En definitiva, venían a reconocer que sólo era preocupante que los extraños pegasen a las mujeres. Claro, como recuerda Cherry (1995), estos autores condujeron su investigación en una época en la que, por una parte, el alcance del maltrato a mujeres no estaba bien documentado y, además, éstas no podían perseguir legalmente a sus maridos por violación. Por otra parte, en el caso que la violencia contra las mujeres fuera tomada como un problema social, siempre se interpretaba desde la perspectiva de la psicopatología, bien del agresor, bien de la víctima.

Así pues, y gracias, en gran medida, a la acción del movimiento feminista durante los años setenta, el marco interpretativo en el que situar la muerte de Kitty Genovese había cambiado lo suficiente como 
para que, en la década de los ochenta, Cherry pudiera apreciar que el suceso tenía que ver con esa temática general que se conoce con el nombre de relaciones de sexo/género. Sin embargo, Cherry (1995) da, aún, un paso más:

"En algún momento, empecé a reconsiderar el asesinato de Kitty Genovese; aunque era un ejemplo de violencia contra las mujeres, tenía también implicaciones más amplias. Señalaba la creciente expresión, por parte de una comunidad, de un sentido de impotencia para prevenir la violencia. El aumento de informes sobre ataques a mujeres también parecía indicar que algunas comunidades eran más vulnerables que otras, por razón de raza y clase social" (p.24)

Es así como Cherry se llegó a interesar por saber más del asaltante de Kitty, Winston Moseley, un hombre negro de casi treinta años, casado con una enfermera y con dos hijos que trabajaba de operario en una factoría. Su abogado defensor dijo de él que había pasado de ser una persona introvertida a convertirse en un maníaco. El juez lo describió como un monstruo. Y los psiquiatras lo consideraron un enfermo mental etiquetado como esquizofrénico catatónico y fue presentado en el juicio como alguien incapaz de detener su inclinación a matar. Condenado inicialmente a la pena de muerte, finalmente se le conmutó por cadena perpetua. Cuatro años después de entrar en prisión se fugó en un traslado. En su huida, raptó y violó a una mujer negra que no lo delató por miedo a que cumpliera su amenaza de matar a sus hijos si lo hacía. A pesar de ello, fue acusada de ayudarlo a escapar y estuvo a punto de ser condenada. Sólo le salvó el hecho que la única mujer negra del equipo del fiscal se negó a imputarla alegando que si se hubiera tratado de una mujer blanca no habría sido lo mismo. Ello le costó el cargo y fue despedida.

Moseley fue al fin detenido y llevado de nuevo a prisión. Pero no acaba aquí la historia del personaje. Trece años después de la muerte de Kitty Genovese, Moseley escribe un artículo en el New York Times dónde, con un estilo ágil e inteligente, explica que ha comprendido que la vida humana es de gran valor, que se ha licenciado en sociología y que el hombre que mató a Kitty Genovese ha dejado de existir. El relato que este hombre hace de su vida además de sobrecogedor es francamente ilustrativo. Hasta tal punto que supone para Cherry un nuevo vuelco en su manera de contemplar el episodio de la muerte de Kitty Genovese. El marco de las relaciones de género se queda pequeño y debe ser ampliado:

"...si permanecemos demasiado focalizados en la violencia hacia las mujeres, perdemos de vista otros factores sistémicos que estrcuturan la violencia -la pobreza, la raza y la clase. Aunque sin absolver a Moseley de su crimen, es preciso que comprendamos el mundo que lo formó en sus primeros años en Detroit y Pittsburg en los treinta y cuarenta (...) La experiencia cotidiana de la violencia en las vidas de la gente es una historia descontextualizada demasiado fácilmente por las teoría psicosociológicas que operan en el nivel conductual individual. Si teorizamos en el nivel de la comunidad, entonces empezamos a tener en cuenta que algunos grupos o comunidades son más vulnerables a la violencia que otros y que esto ha sido así históricamente" (p.27) 


\section{Conclusión: Una visión construccionista}

Esta necesariamente extensa descripción del caso de Kitty Genovese sirve perfectamente como ilustración del argumento que estamos sosteniendo. Por un lado, los actos agresivos no lo son al margen de las colectividades en los que se producen. Por otro, los actores involucrados no tienen un perfil homogéneo sino que se nos muestran llenos de matices y como productos de contextos sociohistóricos concretos. Finalmente, la interpretación que hacemos de todo ello, es decir, la forma que tenemos de ver actos y actores, no es tampoco constante sino contingente y afectada por los recursos interpretativos que están socialmente disponibles. Y sería ingenuo pensar que tales recursos están al margen de los intereses de poder y dominación que atraviesan a toda formación social.

Es en este sentido que una Psicología Social de orientación crítica difícilmente puede estar interesada en estudiar las causas de la agresividad o la violencia como si se tratara de pautas de comportamiento estable y universal, sino que, más bien, vuelca su interés en comprender los procesos por los que las sociedades se dotan de esos recursos interpretativos a los que hemos aludido y cuáles son las consecuencias que se derivan de ello. Es decir, antes que preocuparse por delimitar las características de la acción agresiva o violenta se interesará por las explicaciones que los miembros de una comunidad utilizan para definirla, esto es, se interesará por los discursos en torno a la agresión y a la violencia, incluidos los que emanan de la ciencia como institución social.

Los discursos de la violencia son relativos a tiempos y sociedades específicos que generan modalidades y valores distintos para definir confrontaciones, situaciones, interacciones, causas, etc. Los encontramos siempre imbricados con otros discursos equivalentes sobre la raza, los grupos étnicos o el género, conformando en su totalidad los rasgos definitorios de una cultura. En palabras de Stainton-Rogers et al. (1995):

"No hay una única o simple entidad a la que podamos llamar agresión sino un complejo abanico de diferentes acciones que bajo las circunstancias locales apropiadas llevarán a que se use el término agresión en la explicación" (p.169)

Los discursos que generan ciencias como la sociología o la psicología sobre la agresión y la violencia, son especialmente interesantes en tanto que contribuyen a configurar los criterios mediante los cuales las personas comprenden y analizan estos fenómenos sociales. Es bien sabido, a partir de la obra de Foucault, que la ciencia es un potente dispositivo naturalizador que tiene un papel privilegiado en la operacionalización del control social (Foucault, 1975, 1976). Deconstruir la agresividad y la violencia y mostrarlas como producto de prácticas objetivizantes se convierte, pues, en una precaución necesaria y previa a todo intento de análisis de la cuestión.

\section{Bibliografía}

Bandura,A. (1976) Teoría del aprendizaje social. Madrid: Espasa-Calpe, 1982.

Bandura,A. y Ribes,E. (1975) Modificación de conducta. Análisis de la agresión y la delincuencia. México: Trillas.

Bandura,A., Ross,D. y Ross,S.A. (1963) Imitation of film-mediated agressive models. Journal of Abnormal and Social Psychology, 66:3-11. 
Borofsky,G.; Stollak,G. y Messé,L.(1971) Bystander reactions to physical assault: sex differences in reactions to physical assault. Journal of Experimental Social Psychology 7:313-318.

Brownmiller,S.(1975) Against our will: men, women and rape. New York: Simon and Schuster.

Cherry,F. (1995) The 'stubborn particulars' of social psychology. London: Routledge.

Dollard,J.; Doob,L.; Miller,N.; Mowrer,O. y Sears,R. (1939) Frustration and Aggression. New Haven: Yale University Press.

Domènech,M. e Ibáñez,T, (1998) La Psicología Social como Crítica. Anthropos (En prensa)

Eibel-Eibesfeldt, I. (1973) El hombre preprogramado. Lo hereditario como factor determinante del comportamiento humano. Madrid: Alianza, 1983)

Foucault,M. (1975) Vigilar y castigar. Madrid: Siglo XXI, 1978.

Foucault,M. (1976)Historia de la sexualidad. La voluntad de saber. Madrid: Siglo XXI, 1980.

Freud,S. (1930) El malestar en la cultura. Madrid: Alianza Editorial, 1970.

Gergen,K.J. (1984) Agression as discourse. En A.Mummendey (Ed.) Social Psychology of Aggression. From Individual Behavior to Social Interaction. Berlin: Springer-Verlag. Pp.5168)

Ibáñez,T. e Îñiguez,L. (Eds.) (1997) Critical Social Psychology. London: Sage.

Latané,B. y Darley,J.M. (1970) The unresponsive bystander: Why doesn't he help?. New York: Appleton Century Crofts.

Lorenz, K. (1963) Sobre la agresión. El pretendido mal. México: Siglo XXI, 1981.

Lubek,I. (1979) A brief social psychological analysis of research on aggression in social psychology. En A.R.Buss (Ed.) Psychology in Social Context. New York: Irvington.

Martín-Baró, I. (1983) Acción e ideología. San Salvador: UCA editores, 1996.

Muñoz,J. (1988) Psicología Social de la agresión. Análisis teórico y experimental. Tesis Doctoral. U.A.B.

Muñoz,J. (1990) El papel de las normas en la definición de la agresión. Boletín de Psicología 26:3351.

Shotland,R.L. y Straw,M.K (1976) Bystander response to an assault: when a man attacks a woman. Journal of Personality and Social Psychology 34:990-999.

Stainton-Rogers,R.; Stenner,P.; Gleason,K. y Stainton-Rogers,W. (1995) Social Psychology. A critical agenda. Cambridge: Polity Press.

\section{Referencia}

Doménech,M. e Iñiguez,L. (2002). La construcción social de la violencia. Athenea Digital, 2. Disponible en http://blues.uab.es/athenea/num2/domenech.pdf 